\title{
Drug-Excipient Interaction of Methylphenidate with Glycerin in Methylphenidate Oral Solution and Identification of its Transesterification Products by UPLC-MS/MS
}

\author{
Kishore Kumar Hotha*, Swapan Roychowdhury, Veerappan Subramanian \\ Novel Laboratories Inc., Somerset, NJ, USA \\ Email: "drhotha@gmail.com, khotha@novellabs.net \\ Received 11 December 2015; accepted 30 January 2016; published 2 February 2016 \\ Copyright @ 2016 by authors and Scientific Research Publishing Inc. \\ This work is licensed under the Creative Commons Attribution International License (CC BY). \\ http://creativecommons.org/licenses/by/4.0/ \\ (c) (i) Open Access
}

\begin{abstract}
Reactions between active drug substances and excipients are of interest in the drug formulation process should be checked for the interactions during the storage conditions. Some excipients react with certain chemical groups in drug substances which will form new impurities in the finished product formulations. In the present paper transesterification reaction of methylphenidate with glycerin to form different structural isomeric products was described. These impurities identified in forced degradation studies, excipient compatibility studies and stability analysis of the finished product. Stability samples were analyzed and observed that about $\sim 0.6 \%$ of the Methylphenidate content was transformed into methylphenidate-glycerin isomers within 3 Months at $400^{\circ} \mathrm{C} / 75 \% \mathrm{RH}$ and 18 Months at $25^{\circ} \mathrm{C} / 60 \%$ RH conditions. Analysis of two lots of marketed preparations having expiry dates in 2012 and 2013 showed content of the Methylphenidate esters corresponding to $\sim 0.6 \%$ of the declared Methylphenidate content. The samples of this impurity were investigated by HPLC, UPLC-MS/MS to generate the mechanism of the impurity formation.
\end{abstract}

\section{Keywords}

Methylphenidate, Oral Solution, Glycerin, Transesterification, Excipient Interactions, Forced Degradation

\footnotetext{
${ }^{*}$ Corresponding author.
} 


\section{Introduction}

Methylphenidate (Figure 1) (trade names Concerta, Methylin, Medikinet, Ritalin, Equasym XL, Quillivant XR, Metadate) is a central nervous system (CNS) stimulant of the phenethylamine and piperidine classes that is used in the treatment of attention deficit hyperactivity disorder (ADHD) and narcolepsy. Methylin ${ }^{\circledR}$ (methylphenidate $\mathrm{HCl}$ ) oral solution is a mild central nervous system (CNS) stimulant available as $5 \mathrm{mg} / 5 \mathrm{~mL}$ and $10 \mathrm{mg} / 5 \mathrm{~mL}$ oral solutions for oral administration. Each $\mathrm{mL}$ of Methylin Oral Solution $5 \mathrm{mg} / 5 \mathrm{~mL}$ contains $1 \mathrm{mg}$ of methylphenidate hydrochloride USP. Each $\mathrm{mL}$ of Methylin Oral Solution $10 \mathrm{mg} / 5 \mathrm{~mL}$ contain $2 \mathrm{mg}$ of methylphenidate hydrochloride USP. In addition, Methylin Oral Solution also contains the following inactive ingredients: citric acid anhydrous, glycerin, N\&A grape flavor, PEG 1450, and purified water [1].

Methylphenidate oral solution shows two unknown impurities in the related compound method which is more than the identification threshold as per the ICH guidelines. These impurities identified in forced degradation studies, excipient compatibility studies and stability analysis of the finished product. Manufacturer's impurities and chemical instabilities were checked and identified that these impurities formed due to the drug excipient interaction of the Methylphenidate with glycerin. The objective of the present research article is to give a procedural identification of these unknown peaks and its formation, stability and toxicity in the Methylphenidate oral solution using HPLC, LC-MS/MS. The formation of these impurities is less than one percent, so that the isolation and characterization of these impurities was not feasible. Analysis of the stability samples, drug-excipient compatibility samples and commercially available marketed solutions were performed by LC-MS/MS and HPLC and confirmed the root cause and formation pattern of these impurities.

Methylphenidate and its related impurities were measured using various analytical techniques. Spectrophotometric, RP-HPLC and LCMS methods [2]-[8] gas-chromatographic-mass spectrometric procedures described for quantitation of methylphenidate and ritalinic acid [9]. Analytical method was developed and validated as per the ICH guidelines for the quantification of known and unknown impurities by HPLC.

\section{Experimental}

\subsection{Chemical and Reagents}

Methylphenidate working standard and impurity standards (Figure 1) were supplied by Noramco, Wilmington, USA. The HPLC grade Acetonitrile, methanol, glycerin, acetic acid and analytical grade hexane sulfonic acid sodium salt, ortho phosphoric acid, were purchased from Merck, Darmstadt, Germany. Water used was obtained by using Millipore MilliQ Plus water purification system. Drug product samples were supplied by Novel Laboratories Inc., Somerset, New Jersey.

\subsection{Equipment}

Water HPLC with 2695 separation module equipped with 2996 PDA detector used for the HPLC analysis. Acquity UPLC-MS/MS was used for the identification of the unknown impurities. The output signal was monitored and processed using empower 3 software. Cintex digital water bath was used for hydrolysis studies. Thermal stability studies were performed in a dry air oven (Cintex, Mumbai, India).

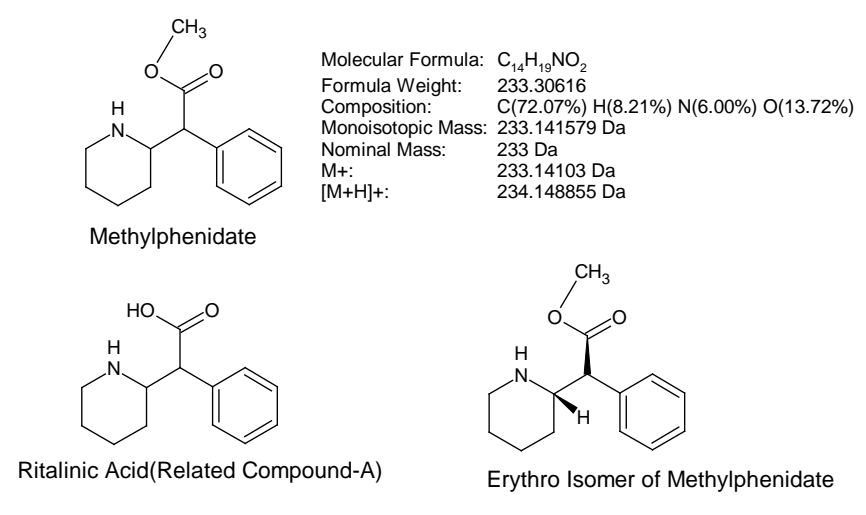

Figure 1. Chemical structures of methylphenidate and impurities. 


\subsection{Chromatographic Conditions}

The chromatographic column used was a Symmetry Shield RP 18, $150 \times 4.6 \mathrm{~mm}, 5.0 \mu \mathrm{m}$. The separations was achieved on a gradient method. Solvent A is a mixture of Buffer: Acetonitrile (92:8(V/V) [Buffer: $6.8 \mathrm{~g}$ of monobasic potassium phosphate and $2.0 \mathrm{~g}$ of Sodium 1-octane sulfonate monohydrate in $1000 \mathrm{~mL}$ water adjusted to $\mathrm{pH} 2.2 \pm 0.05$ ] and solvent B is mixture of Acetonitrile and THF in the ration (95:5). The flow rate was 1.5 $\mathrm{mL} \cdot \mathrm{min}^{-1}$ and the detection wavelength was $265 \mathrm{~nm}$. The HPLC gradient program was set as: Time (min)/\% solution B: 00/15, 8/15, 40/40, 41/15, 50/15. The column temperature was maintained at $40^{\circ} \mathrm{C}$ and the injection volume was $20 \mu \mathrm{L} .1 \%$ phosphoric acid was used as a Diluent.

\subsection{Liquid Chromatography and Mass Spectrometric Conditions}

LC-MS/MS system (Acquity UPLC coupled with TQD mass spectrometer with empower software, Waters Corporation, Milford, USA) was used for the identification of unknown compounds formed during forced degradation and stability testing studies. The method was developed using Acquity UPLC BEH C18 $1.7 \mu \mathrm{m}, 2.1 \times$ $150 \mathrm{~mm}$ column as a stationary phase. The mobile phase A is a $0.1 \%$ of acetic acid in water. The mobile phase B is acetonitrile. The UPLC gradient program was set as: Time $(\min ) / \%$ solution B: 00/15,5.58/15,26.92/ $40,26.98 / 15,35.00 / 15$. The column temperature was maintained at $40^{\circ} \mathrm{C}$ and the injection volume was $15 \mu \mathrm{L}$. Milli-Q water was used as Diluent. The mobile phase pumped at $0.64 \mathrm{~mL} / \mathrm{min}^{-1}$. The eluted compounds were monitored at $210 \mathrm{~nm}$. The run time was $35 \mathrm{~min}$. Mass spectrometric conditions optimized as cone gas $10 \mathrm{~V}$, Collision flow $70 \mathrm{~L} / \mathrm{Hr}$., Ion energy 0.7 Entrance and Exit Potentials $1 \mathrm{~V}$, source temperature $150^{\circ} \mathrm{C}$, Desolovation gas $800 \mathrm{~L} / \mathrm{hr}$., Desolvation temperature $400^{\circ} \mathrm{C}$.

\subsection{Forced Degradation Studies}

Analytical method developed and validated on HPLC which separates all the impurities (Figure 2) from the Methylphenidate and excipients used in the formulation. This method was tested for accuracy, precision, linearity, specificity and found stability indicating [9]-[12]. The representative chromatogram of the impurity solution was shown in Figure 2. Forced degradation studies were also performed on Methylphenidate oral solution using these chromatographic conditions. The stress conditions employed for degradation study includes light (carried out as per ICH Q1B), Dry heating done at $80^{\circ} \mathrm{C}$ for about 4 hrs., acid hydrolysis (with $1 \mathrm{~N} \mathrm{HCl}$ solution for about $2 \mathrm{Hrs}$ at $80^{\circ} \mathrm{C}$ ), base hydrolysis (with $1 \mathrm{~N} \mathrm{NaOH}$ solution for about $0.3 \mathrm{Hrs}$ at $\mathrm{RT}$ ), water hydrolysis and oxidation (Treated with $3 \%$ Hydrogen peroxide $\left(\mathrm{H}_{2} \mathrm{O}_{2}\right)$ for about $4 \mathrm{Hrs}$ at $60^{\circ} \mathrm{C}$ ). Sunlight, thermal and UV degradation were also performed and purity of stressed samples was checked by using Photo diode array detector (PDA). The purity factor is within the threshold limit obtained in all stressed samples demonstrates the analyte peak homogeneity.

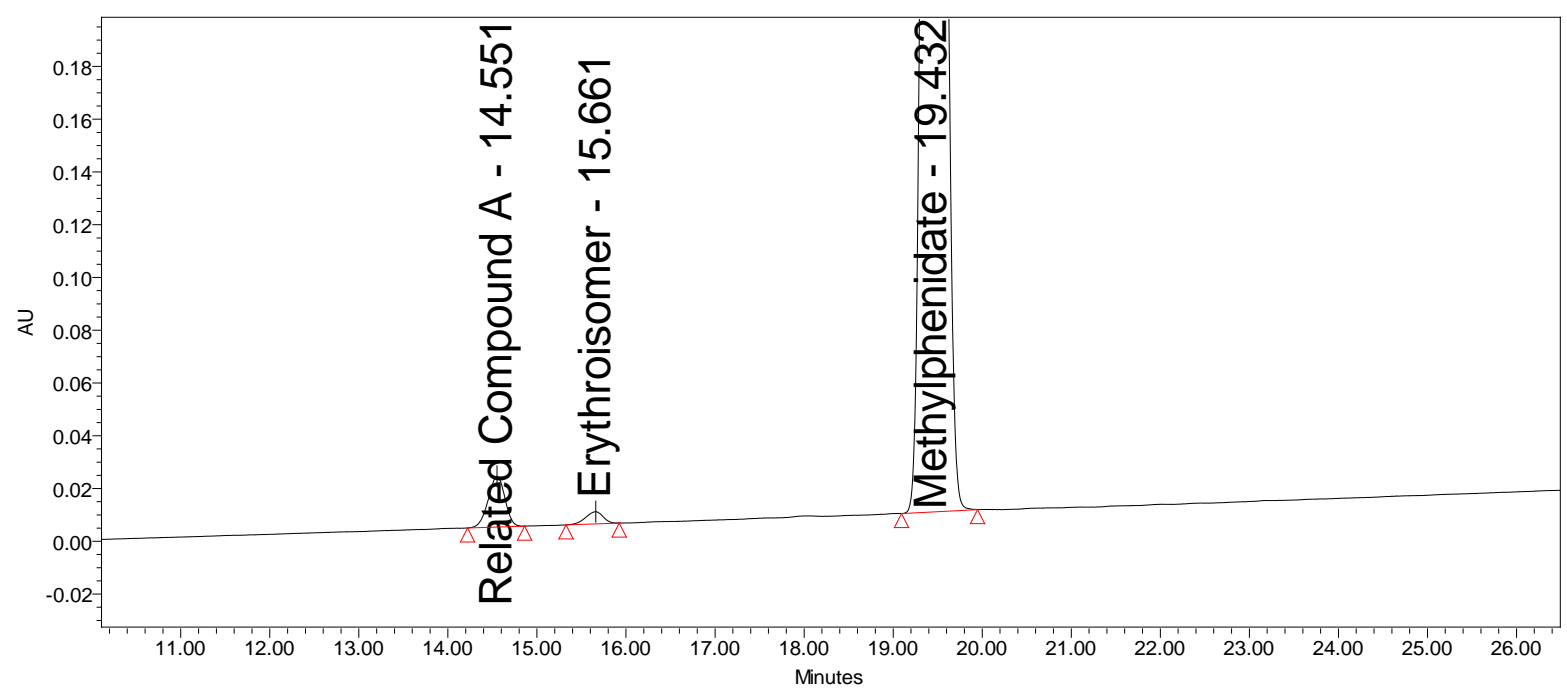




\subsection{Excipient Compatibility Studies and Generation of Reaction Products}

Analytical methodology described in the section 2.3 was used for the analysis of compatibility studies. Compatibility studies were performed for all the excipients as per the composition of finished product. All the utilized individual excipients were taken as per the composition with drug and exposed to temperature at $80^{\circ} \mathrm{C}$ for 16 Hours. After the specified time, volumetric flasks were withdrawn from hot air oven and allowed to reach room temperature. After reaching room temperature, samples were diluted with diluent up to $20 \mathrm{~mL}$. All the samples were injected as per the method.

\subsection{Quantitative Analysis and Stability of the Finished Product}

Stability sample analysis of methylphenidate oral solution was performed using method described in the section 2.3. Accelerated samples for Initial, 1,2 and 3 months and controlled room temperature stability samples (initial, 3,6,9,12,18 and 24 months) were analyzed. The quantitative measurements of these esters formed in the finished product measured using validated analytical method. Due to the non-availability of the reference standards for the methylphenidate-glycerin esters, Quantitation was performed by using methylphenidate standard assuming that the same molar absorbance for both active and the formed impurities. For erythro isomer and ritalinic acid respective standards were used to quantify these impurities in the finished product.

\subsection{Commercial Liquid Preparations of Methylphenidate}

Two lots of methylphenidate preparations were purchased. Both preparations are $5 \mathrm{mg} / 5 \mathrm{~mL}$ contains methyl phenidate, citric acid anhydrous, glycerin, N\&A grape flavor, PEG 1450, and purified water. The expiry dates of these preparations were 11/2012, 5/2013.

\subsection{Mass Spectrometric Analysis}

To carry forward the structural elucidation of the unknown impurity Mass spectrometric analysis was performed. Identical UPLC method developed based on the HPLC of Methylphenidate related substance method due to the ion pair buffer in the HPLC method of analysis. Elution patterns were compared by developing an UPLC-MS/ MS method with volatile buffer and found sufficient separation of the impurity of interest.

\subsection{Toxicology-Mutagenicity Endpoint}

The toxicology and mutagenicity endpoint of the formed Methylphenidate-glycerin esters were evaluated using the Lasha Sarah Nexus Trail software version 1.1.2 which will give the mutagenicity endpoint as per the ICH M7 guidelines.

\section{Results and Discussion}

\subsection{Development of Identical UPLC-MS/MS Method}

There were several tailbacks in the process to identify the structure of the impurity. Analytical method was developed using ion pair buffer because of the hydrophilic nature of the Methylphenidate and also for the separation of 6 impurities from the main peak. In the reversed-phase chromatography nitrogen compounds were attached to chemically bonded silica which gives peak tailing, poor separation and reproducibility problems. These problems are probably due to the presence of residual silanol groups on the surface of the column material. 1-Hexane sodium sulfonate (anionic ion pairing agent) was added to the mobile phase. The ion-pair reagent is attracted to the stationary phase because of its hydrophobic alkyl group, and the charge carried by the reagent $\left(\mathrm{C}_{6}-\mathrm{SO}^{3-}\right)$ there by attaches to the stationary phase. This negative charge on the stationary phase is balanced by positive ions $\left(\mathrm{Na}^{+}\right)$from the reagent. A positively charged sample ion (protonated base) can now exchange with $\mathrm{Na}^{+}$ion, resulting in the retention of the sample ion by an ion exchange process. By this way hexane sulfonate adjusts the retention and improves the resolution.

In order to run the samples in LC-MS/MS method, it was decided to develop a method compatible for mass spectrometry. The ion pair buffer and phosphate buffer used in the HPLC method was not compatible with UPLC-MS/MS. So that UPLC method was developed using volatile buffer in the mobile phase. $0.1 \%$ formic 
acid in water was used as a mobile phase to match with the lower pH of HPLC buffer. Electrospray Ionization of positive ion mode was selected for the mass spectrometric analysis. $0.1 \%$ formic acid in water was used as a diluent instead of $0.1 \%$ phosphoric acid. Same Identical Pattern was reproduced using the above conditions. To identify the unknown impurities in the UPLC method, spectral characteristics were compared and observed that there is no significant change in the UV Spectra of impurities in consideration with other peaks. To confirm the unknown peaks of interest, elution pattern and \% area of the unknown peaks in HPLC (Figure 3(a)) were compared in UPLC-MS/MS method and identified the unknown peaks eluted at 2.29 and 2.38 (Figure 3(b)).

\subsection{Observations and Structural Elucidation by Mass Spectrometry}

The unknown peaks were observed in the excipient compatibility studies, when glycerin was added to methylphenidate (Figure 4). The relative retention times of these impurities observed at 0.75 and 0.77 . The remaining impurities, Ritalinic acid and erythro isomer were formed at the relative retention times of 0.80 and 0.84 . In all the other excipients there were no traces of these impurities at that specified retention time which strengthens the

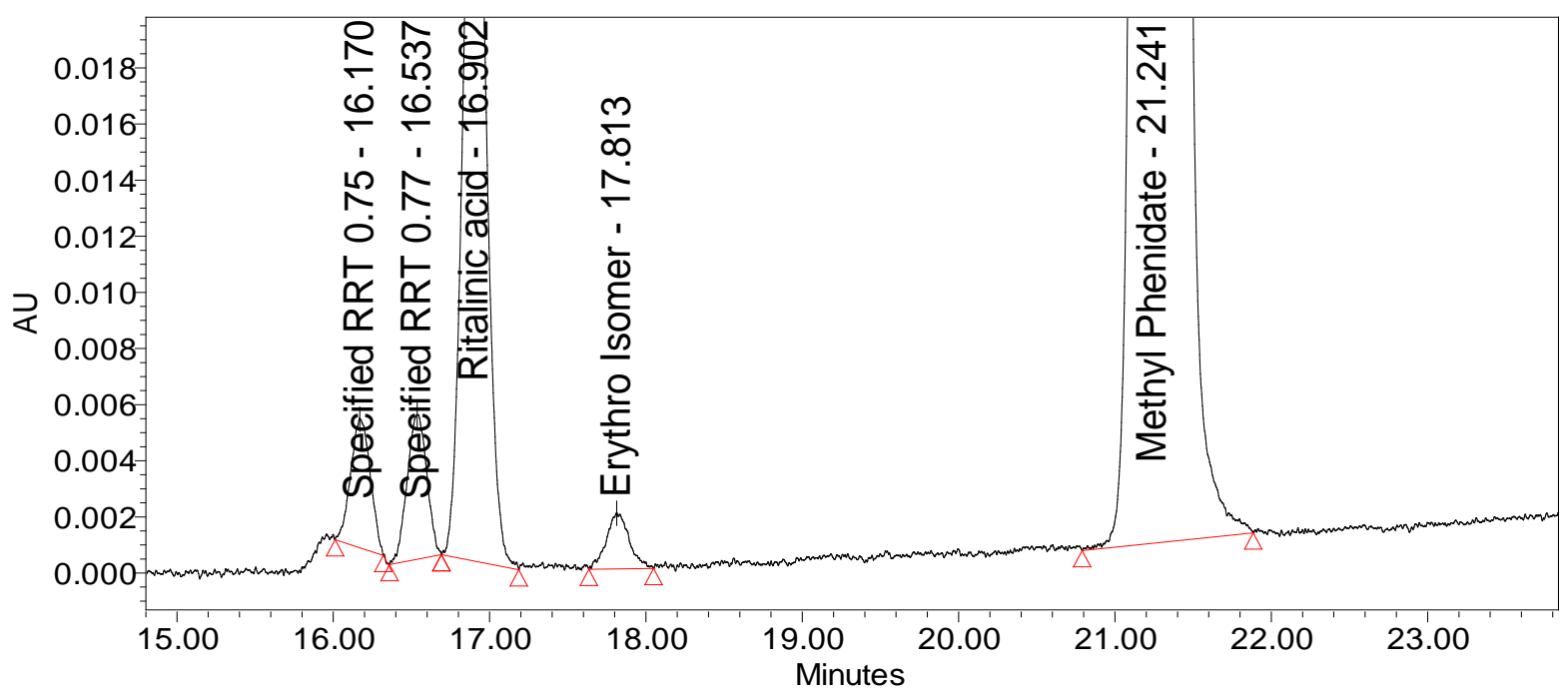

(a)

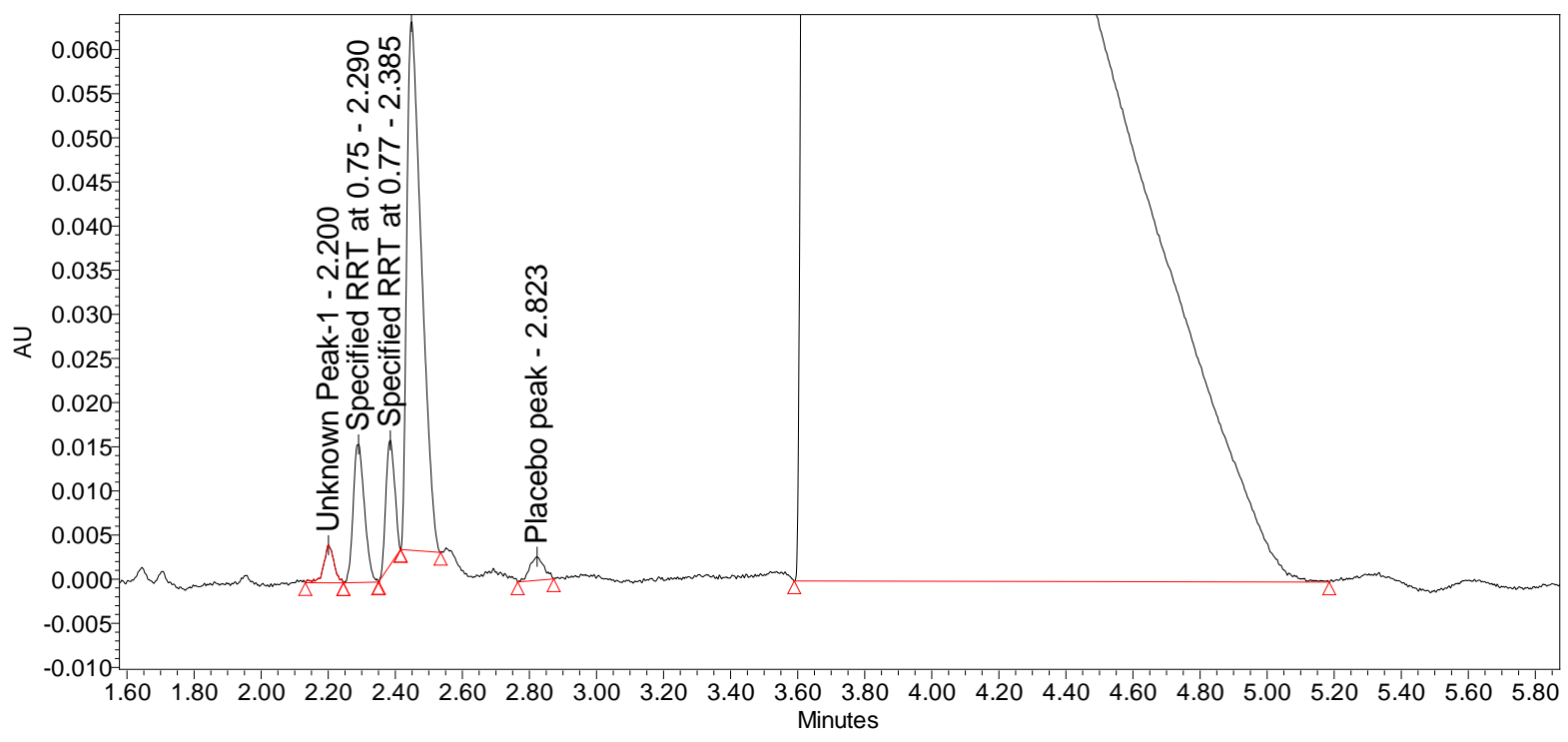

(b)

Figure 3. (a) HPLC Chromatogram of 24 Months CRT Sample; (b) UPLC Chromatogram of 24 Months CRT Sample. 


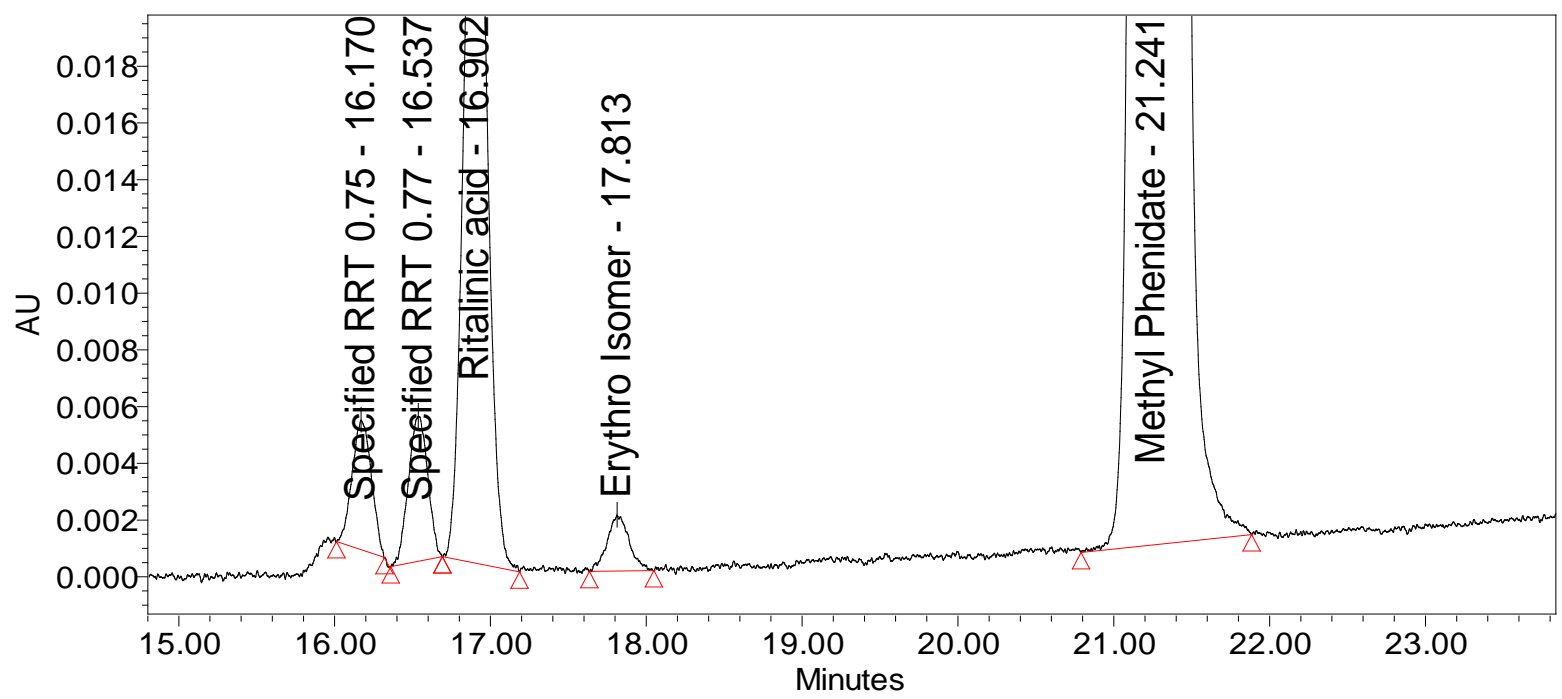

Figure 4. HPLC Chromatogram of methylphenidate and Glycerin at $80^{\circ} \mathrm{C}$ for $16.0 \mathrm{Hrs}$.

formation of the impurities is due to the Glycerin. Spectral characteristics of the impurity found in Methyl- phenidate oral solution at RRT 0.75 and 0.77 min was exactly matches with the impurity observed in the glycerin excipient compatibility experiment. Molecular ion $[\mathrm{M}+\mathrm{H}]^{+}$shows intense ion of 294 confirms the addition of glycerin moiety with Methylphenidate (Figure 5 \& Figure 6). Methylphenidate stability samples, glycerin reacted samples and commercial samples of methylphenidate finished product were analyzed by UPLC-MS/ MS and confirmed the same $\mathrm{M}+\mathrm{H}$ for the impurity formed at 0.75 and 0.77 .

\subsection{Structural Identification of the Possible Isomers Due to Transesterification}

Glycerin (Figure 7) is a trihydric alcohol and, like other alcohols, forms esters, ethers. Amines, aldehydes, and compounds analogous to metallic alcoholates. Structurally, glycerin has two primary and one secondary hydroxyl groups. The primary hydroxyl groups generally more reactive than the secondary group and, of the two primary groups, the first to react usually does so more readily than the second. In any reaction, however, the second and third hydroxyls will react to some extent before all the most reactive groups are exhausted. Reaction mixtures thus contain isomers and products of different degrees of reaction, with the relative amounts of each reflecting their ease of formation.

There were several articles described the formation of glycerin transesterification products in which glycerin is tend to form 1-mono and 2-mono esters when reacts with esters [13]-[17]. The detailed mechanism for the formation of methylphenidate-glycerin esters were described in (Figure 8) [14]. The reaction of primary and secondary alcohols of glycerin with methylphenidate could be the possibility for the formation of these two impurities. Methyl phenidate has two chiral centers. There could be 4 stereo isomers were formed according to the $2^{\mathrm{n}}$ rule (Figure 9). Methylphenidate exists as dl threo and dl erythro forms. Commercially available methyl- henidate is dl threo form. DL erythro isomer is the impurity observed in the synthetic process and in the stability conditions. The four isomers reacts with glycerin to form esters but practically dl threo methylphenidate esters could not be separated using HPLC conditions (Figure 9). There could be a chance for the formation of additional peaks due to the erythro isomer reacts with glycerin to form methylphenidate esters. The formation of erythro isomer is very insignificant so that the reaction of glycerin is difficult to form significant amount of the impurity in the finished product. The formed esters are the regiomers of the glycerin when primary and secondary hydroxyl groups reacts with threo methylphenidate Based on the above observations it was confirmed that the impurities formed in the methylphenidate oral solution is due to the interaction of glycerin with methyl phenidate. Methyl phenidate degrades to Ritalinic acid and Erythro isomer whereas in presence of glycerin it forms Ritalinic acid, Erythro isomer and specified impurity at 0.75 RRT and specified impurity at 0.77 RRT. The collection and characterization of these impurities is very difficult due to the formation of low levels in the finished product. 

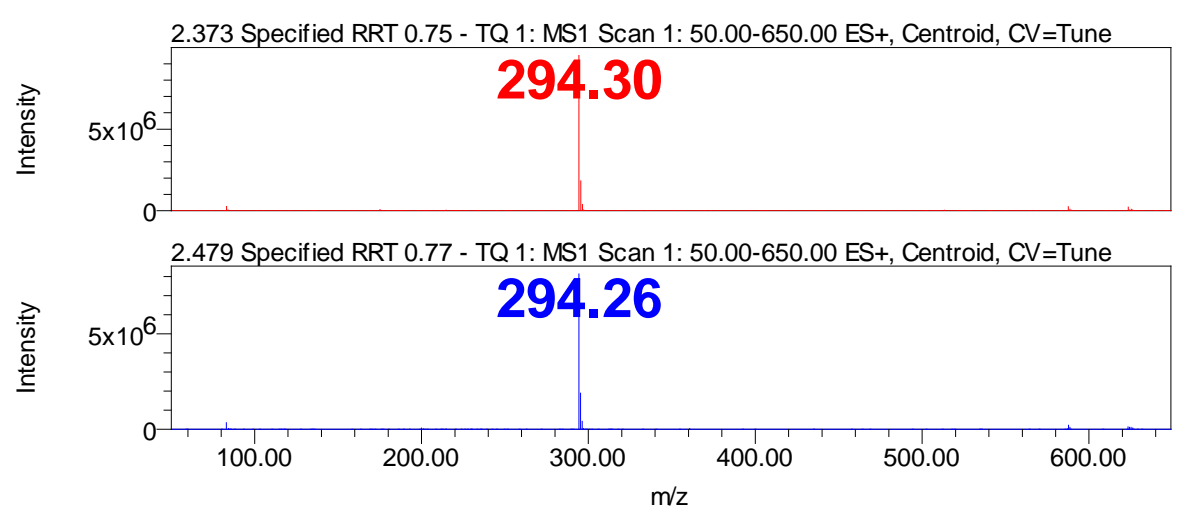

(a)
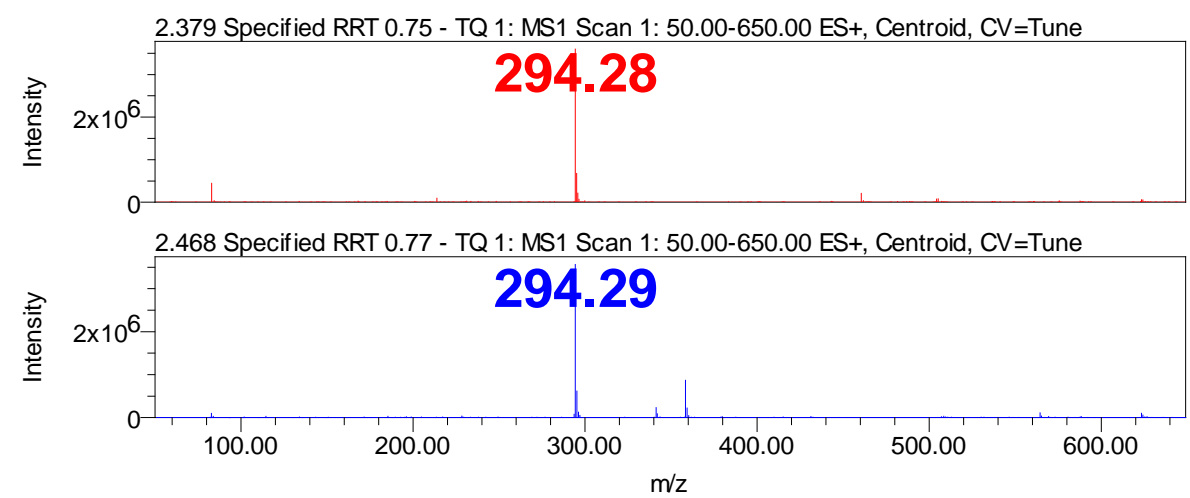

(b)
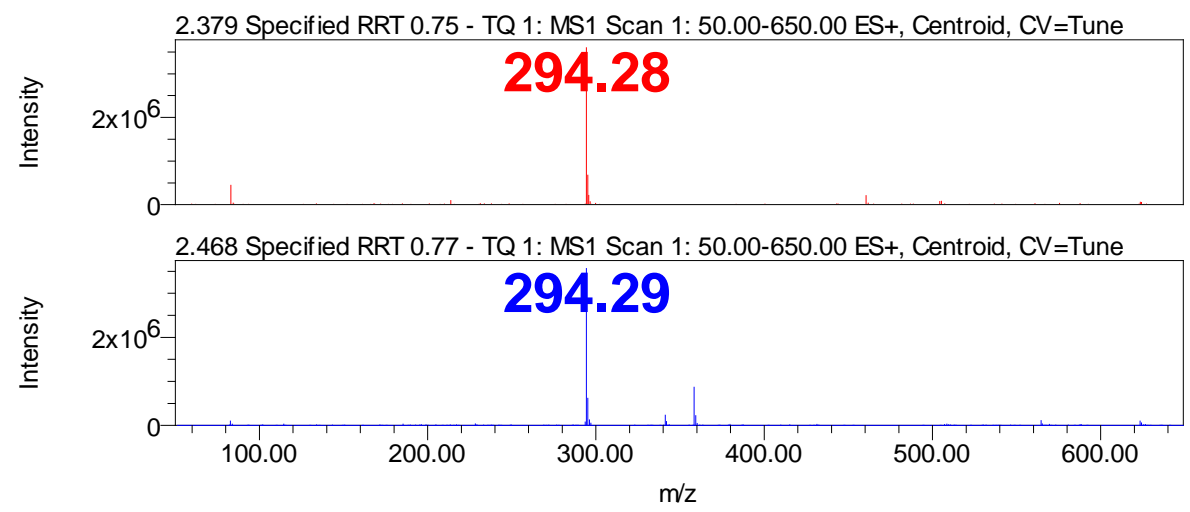

(c)

Figure 5. MS Spectrums of Methylphenidate oral solution samples. (a) Brand sample MS Spectrum; (b)In-House Sample MS Spectrum; (c) Glycerin-Methylphenidate interaction Impurity-MS Spectrum.

\subsection{Forced Degradation Studies}

Forced degradation of the finished product shows the methylphenidate-glycerin esters in acid, base conditions. The formation of the ritalinic acid is the major impurity in all forced degradation conditions. About 1\% Methylphenidate esters found in acid degradation@80 $\mathrm{C} / 2$ Hrs. and $2 \%$ of the esters found in Base degradation @ Room temperature/30 min. Based on the forced degradation results, methylphenidate degrades rapidly in Base hydrolysis. The formation of Erythro isomer is very minimal in all the stressed conditions. The chromatograms and results described in Figure 10, Table 1 and Table 2. 


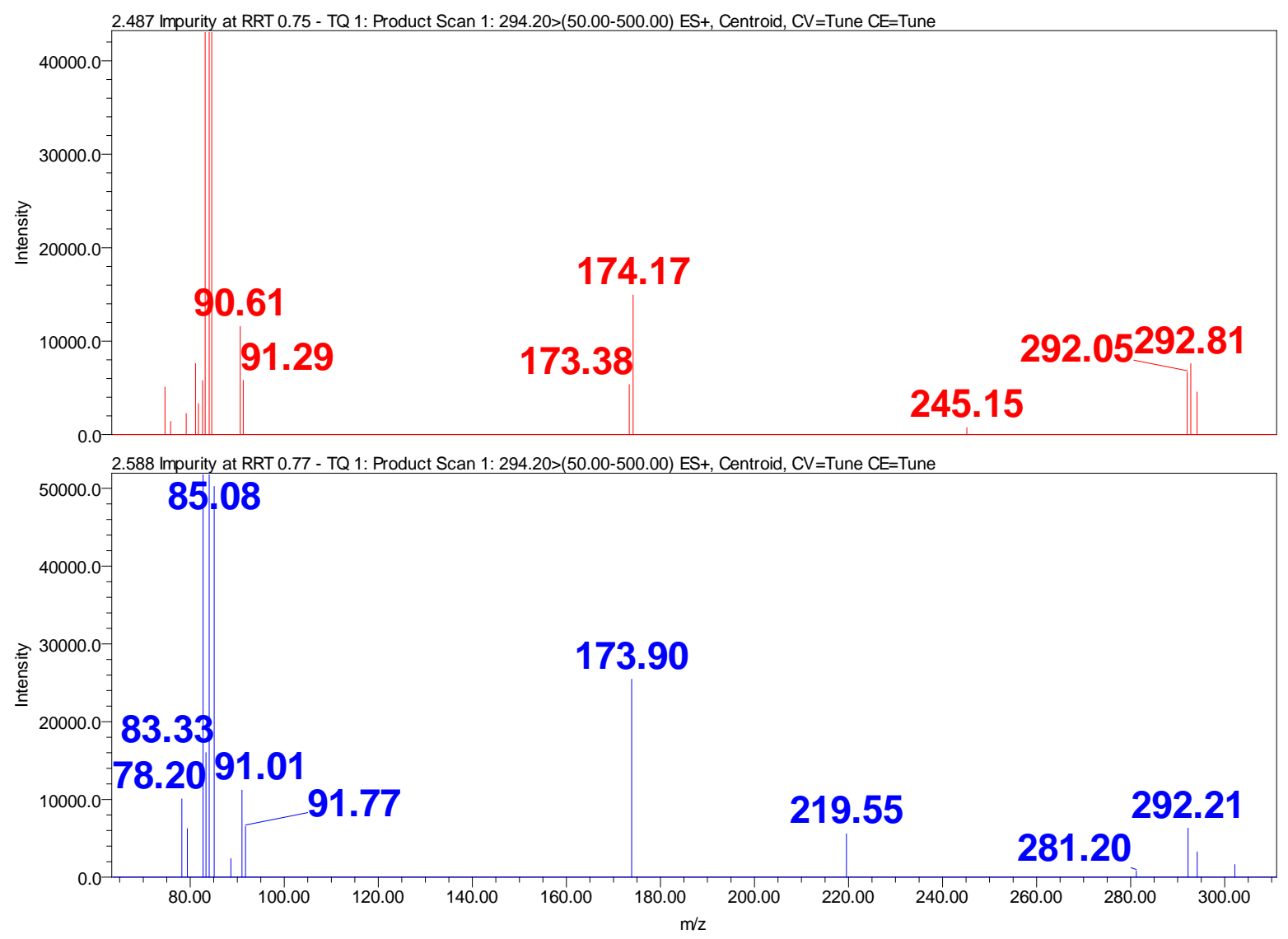

Figure 6. Product ion chromatogram of the methylphenidate-glycerin esters.

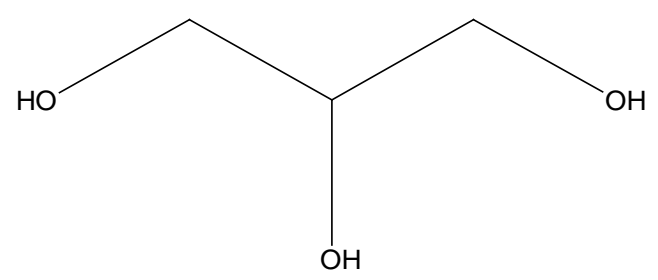

Molecular Formula: $\mathrm{C}_{3} \mathrm{H}_{8} \mathrm{O}_{3}$

Formula Weight: $\quad 92.09382$

Composition: $\quad \mathrm{C}(39.13 \%) \mathrm{H}(8.76 \%) \mathrm{O}(52.12 \%)$

Monoisotopic Mass: $92.047344 \mathrm{Da}$

Nominal Mass: $\quad 92 \mathrm{Da}$

$\mathrm{M}+$ :

$[\mathrm{M}+\mathrm{H}]+: \quad \quad 93.054621 \mathrm{Da}$

Figure 7. Glycerin molecular structure.

Table 1. Forced degradation data for methylphenidate hydrochloride finished product.

\begin{tabular}{|c|c|c|c|c|}
\hline Stress Condition & Reaction Time/Conditions & Purity Angle & Purity Threshold & $\%$ Deg \\
\hline Control Sample-1 & \multirow{2}{*}{ NA } & 0.055 & 0.290 & \multirow{2}{*}{ NA } \\
\hline Control Sample-2 & & 0.050 & 0.282 & \\
\hline Acid Degradation & $5.0 \mathrm{~mL}$ of $1 \mathrm{~N} \mathrm{HCl} / 80^{\circ} \mathrm{C} / 2$ hour & 0.060 & 0.292 & 15 \\
\hline Base Degradation & $5.0 \mathrm{~mL}$ of $0.1 \mathrm{~N} \mathrm{NaOH} / \mathrm{RT} / 30$ minutes & 0.057 & 0.281 & 33 \\
\hline Oxidation & $5.0 \mathrm{~mL}$ of $3 \% \mathrm{H}_{2} \mathrm{O}_{2} / 80^{\circ} \mathrm{C} / 4$ hours & 0.075 & 0.322 & 1 \\
\hline Thermal & Thermal $/ 80^{\circ} \mathrm{C} / 4$ hours (Use water bath) & 0.045 & 0.280 & 1 \\
\hline UV Light & UV Light/24 hours & 0.064 & 0.308 & 0 \\
\hline Spiked Sample & NA & 0.098 & 0.331 & NA \\
\hline
\end{tabular}




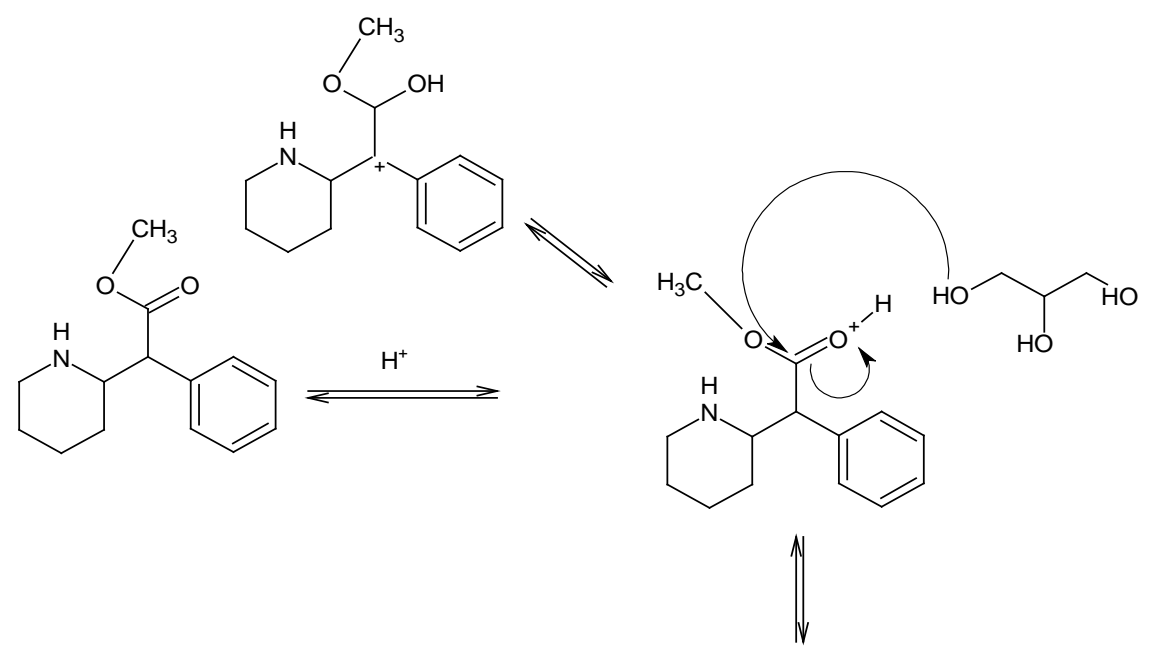<smiles>N#CC1CCCCN1C(C(=O)OCC(O)CO)c1ccccc1</smiles>
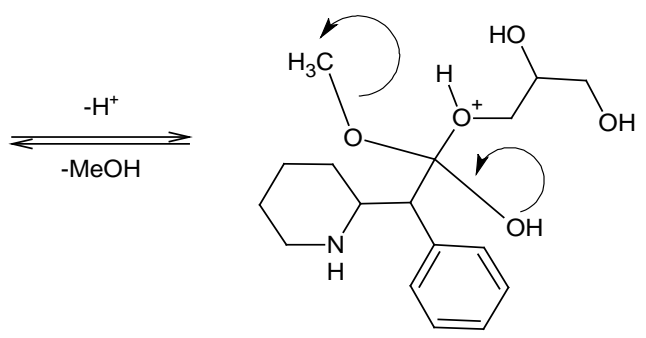<smiles>O=C(OC(CO)CO)C(c1ccccc1)C1CCCCN1</smiles>

Figure 8. Mechanism for the formation of methylphenidate-glycerin esters.

Table 2. Major degradants for methylphenidate hydrochloride finished product.

\begin{tabular}{|c|c|c|c|c|}
\hline Stress Condition & Name of the Peak & RT & RRT & \% Area \\
\hline \multirow{4}{*}{ Base Degradation } & Glycerin Ester- 1 & 13.72 & 0.70 & 3.63 \\
\hline & Glycerin Ester- 2 & 14.15 & 0.72 & 2.9 \\
\hline & Related Compound A & 14.66 & 0.75 & 20.4 \\
\hline & Erythroisomer & 15.77 & 0.81 & 1.42 \\
\hline \multirow{4}{*}{ Acid Degradation } & Glycerin Ester- 1 & 13.68 & 0.70 & 1.00 \\
\hline & Glycerin Ester- 2 & 14.11 & 0.72 & 0.78 \\
\hline & Related Compound A & 14.62 & 0.75 & 12.36 \\
\hline & Erythroisomer & 15.72 & 0.81 & 0.34 \\
\hline \multirow{2}{*}{ Peroxide Degradation } & Related Compound A & 14.7 & 0.75 & 0.14 \\
\hline & Erythroisomer & 15.75 & 0.81 & 0.02 \\
\hline Thermal Degradation & Related Compound A & 14.53 & 0.75 & 0.18 \\
\hline
\end{tabular}



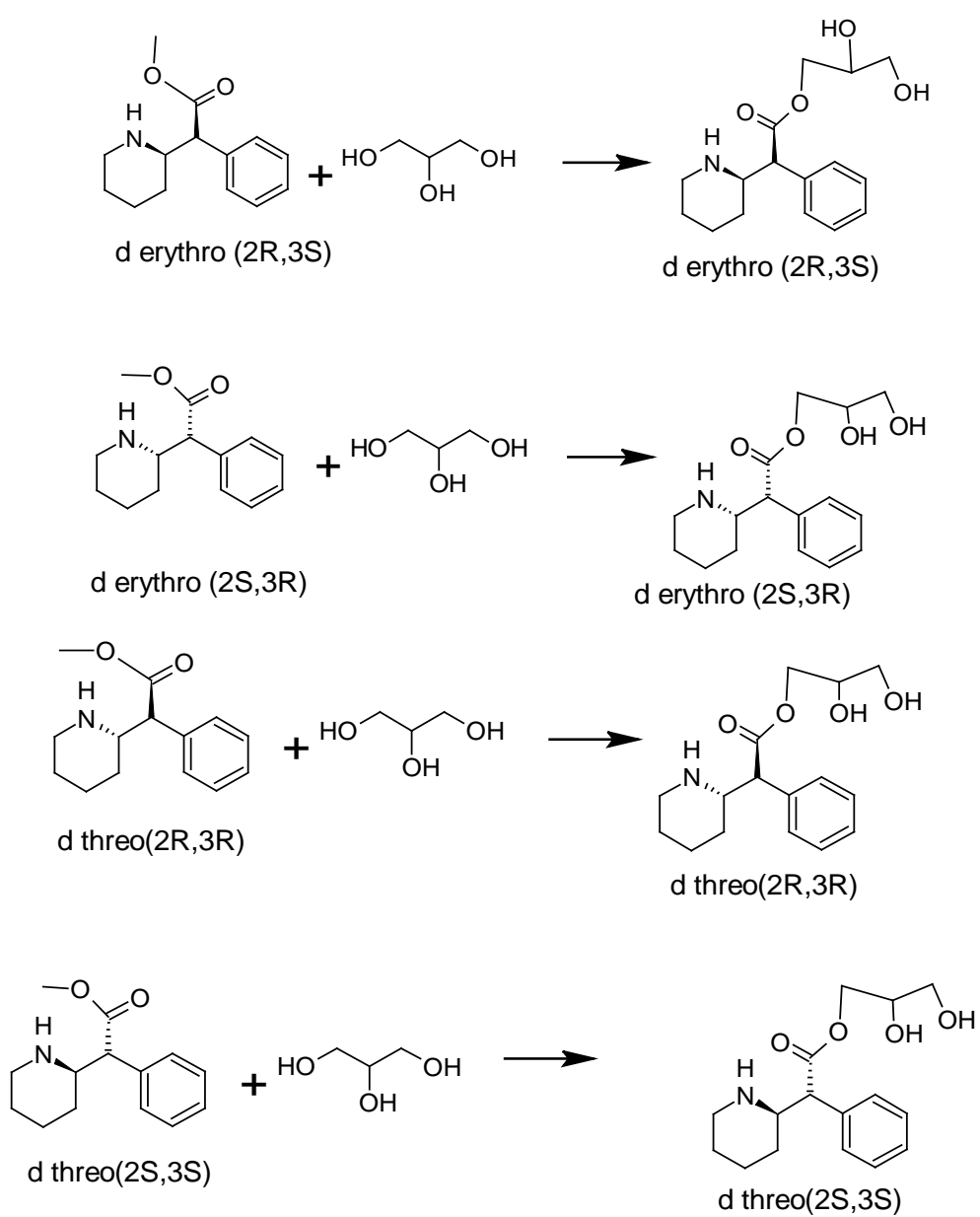

Figure 9. Possible formations of methylphenidate-glycerin esters (Erythro and Threo form).

Table 3. Stability analysis summary of methylphenidate oral solution in CRT and ACC conditions.

\begin{tabular}{|c|c|c|c|c|}
\hline \multicolumn{5}{|c|}{$40^{\circ} \mathrm{C} / 75 \%$ RH Stability Summary } \\
\hline Condition & Ritalinic acid & Erythro isomer & Ester-1 (RRT 0.75) & Ester-2 (RRT 0.77) \\
\hline Initial & 0.00 & 0.00 & 0.00 & 0.01 \\
\hline 1 Month & 0.40 & 0.00 & 0.055 & 0.06 \\
\hline 2 Months & 0.797 & 0.092 & 0.106 & 0.118 \\
\hline 3 Months & 1.301 & 0.136 & 0.164 & 0.162 \\
\hline \multicolumn{5}{|c|}{$25^{\circ} \mathrm{C} / 60 \%$ RH Stability Summary } \\
\hline Initial & 0.00 & 0.00 & 0.00 & 0.00 \\
\hline 3 Months & 0.277 & 0.00 & 0.00 & 0.00 \\
\hline 6 Months & 0.562 & 0.00 & 0.101 & 0.081 \\
\hline 9 Months & 0.768 & 0.098 & 0.142 & 0.087 \\
\hline 12 Months & 1.102 & 0.112 & 0.168 & 0.16 \\
\hline 18 Months & 1.444 & 0.125 & 0.241 & 0.198 \\
\hline 24 Months & 2.061 & 0.172 & 0.348 & 0.318 \\
\hline
\end{tabular}



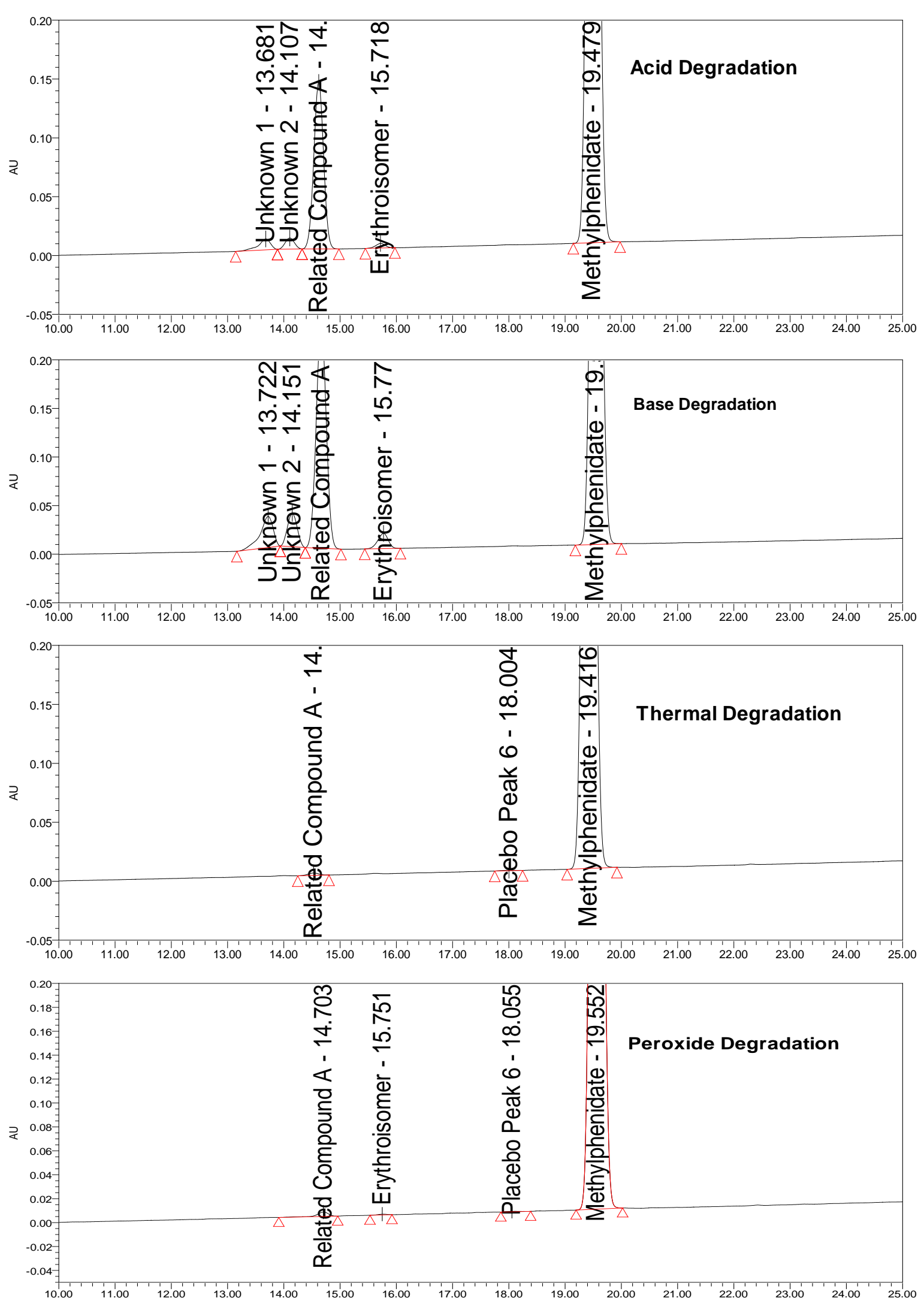

Figure 10. Forced degradation chromatograms of methylphenidate. 


\subsection{Quantitative Analysis and Stability of the Finished Product}

Quantitative analysis of finished product was performed in CRT (Controlled Room Temperature) and accelerated conditions. The formation of the impurity is linear and doesn't degrades after the formation. The results were extrapolated in the graph described in Figure 11. The major impurity observed in the finished product is ritalinic acid with $1.3 \%$ in 3 Months of the accelerated conditions whereas $2.01 \%$ in 24 months of the CRT Conditions. The formation of methylphenidate-glycerin esters at 0.75 and 0.77 RRT were $0.31 \%$ and $0.30 \%$ in three months of accelerated conditions and $0.34 \%$ and $0.31 \%$ in 24 months of CRT conditions (Table 3).

\subsection{Commercial Liquid Preparations of Methylphenidate}

Two lots of commercial liquid preparations of methylphenidate with different expiry dates were analyzed (Figure 12) for the possible content of methylphenidate-glycerin esters at RRT 0.75 and 0.77 . The glycerin esters of methyl phenidate were found in both the lots. This is what expected from the knowledge of the ingredients of the preparations. The conversion percentage of Methylphenidate into the esters was estimated using the peak area of the extracted chromatogram. Quantitative analysis confirms $0.34 \%$ and $0.23 \%$ in 2013 expired lot and $0.34 \%$ and $0.31 \%$ in 2012 expired lot.

\subsection{Toxicology-Mutagenicity Endpoint}

The formed products of these methylphenidate-glycerin ester structures were evaluated using lasha Sarah nexus trail software for the mutagenicity. The Mutagenicity endpoint of these two compounds (Figure 13) is predicted to be negative with $68 \%$ confidence for 1 -mono ester and negative with $73 \%$ confidence for the 2 -mono ester of methylphenidate.

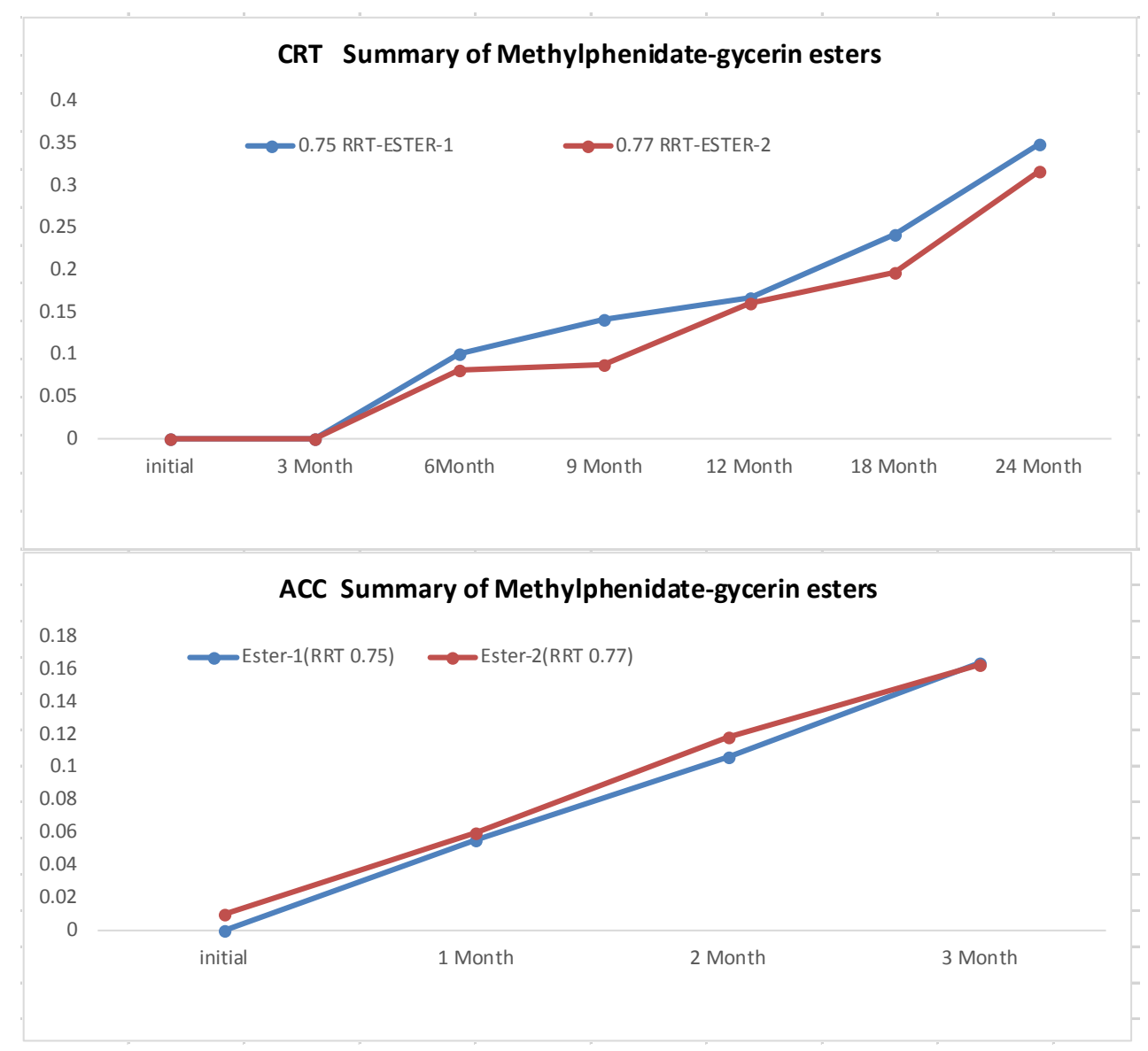

Figure 11. Stability analysis of esters formed with glycerin and methylphenidate. 

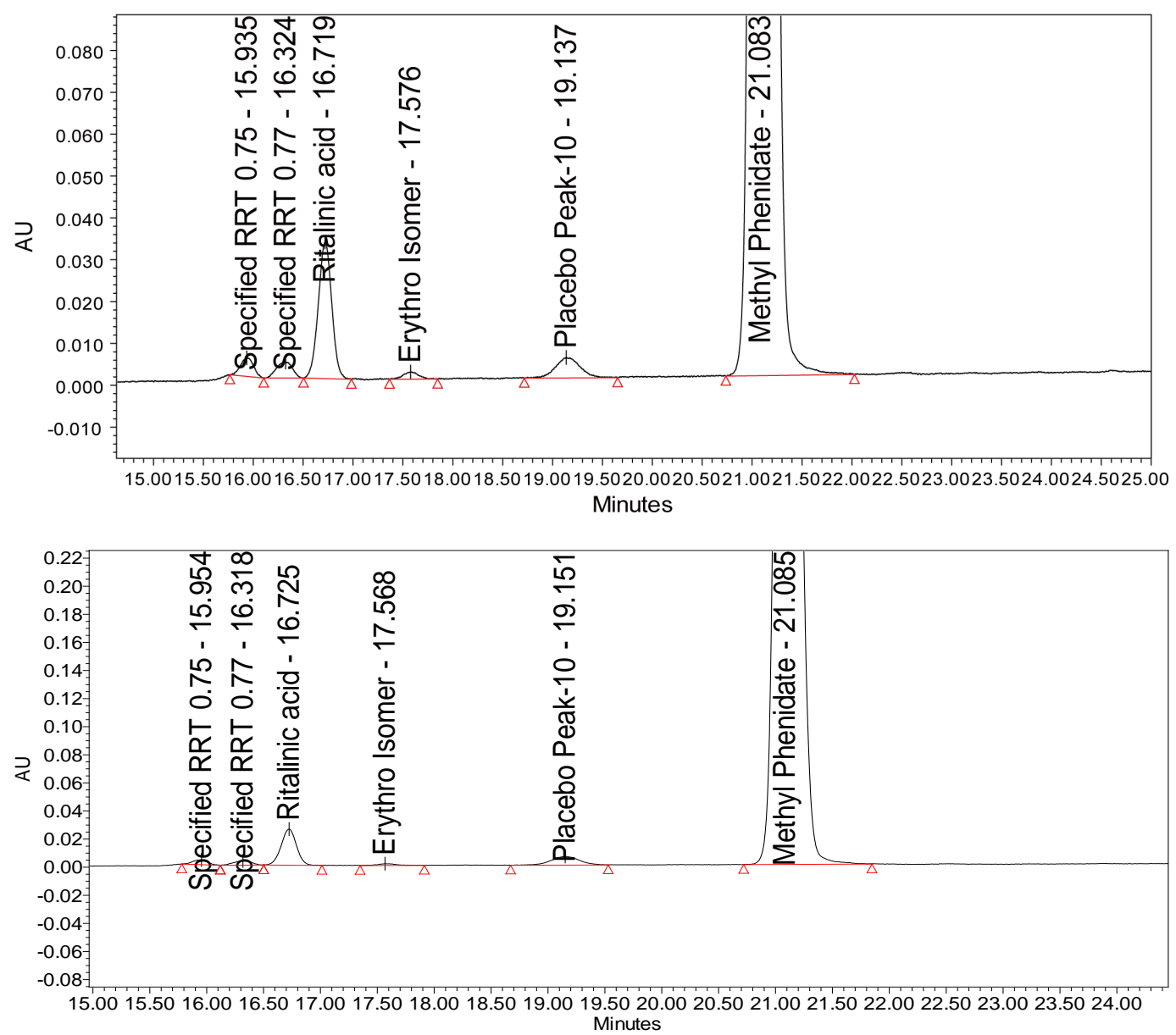

Figure 12. HPLC Chromatogram of the commercially available methylphenidate solution (Expiry 05/2013) \& (Expiry 11/2012).<smiles>O=C(OCC(O)CO)C(c1ccccc1)C1CCCCN1</smiles>

Molecular Formula: $\mathrm{C}_{16} \mathrm{H}_{23} \mathrm{NO}_{4}$ Formula Weight: $\quad 293.35812$

Composition: $\quad \mathrm{C}(65.51 \%) \mathrm{H}(7.90 \%) \mathrm{N}(4.77 \%) \mathrm{O}(21.82 \%)$

Monoisotopic Mass: 293.162708 Da

Nominal Mass: $293 \mathrm{Da}$

$\mathrm{M}+$ :

293.16216 Da

$[\mathrm{M}+\mathrm{H}]+$ :

294.169985 Da<smiles>O=C(OC(CO)CO)C(c1ccccc1)C1CCCCN1</smiles>

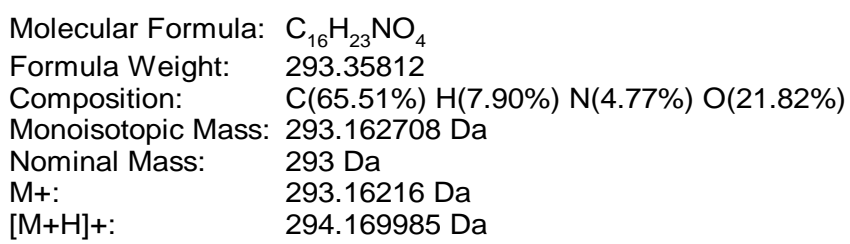

Figure 13. Structures of methylphenidate-glycerin esters. 


\section{Conclusion}

It has been shown that the drug substance methylphenidate reacts with glycerin to form esters. Results of the stability samples, forced degradation studies and excipient compatibility studies confirmed these esters. The formed esters are above the identification limits based on the maximum daily dose of the methylphenidate. Mutagenicity of these esters found negative up to 75\%. Commercially available marketed preparations confirm the formation of these impurities $0.6 \%$.

\section{Acknowledgements}

The authors wish to thank the management of Novel Laboratories INC for providing the infrastructure for the supporting of this research work. Cooperation from colleagues Quality Control and Analytical Research\& Development of Novel Laboratories is appreciated.

\section{References}

[1] https://en.wikipedia.org/wiki/Methylphenidate

[2] Hsieh, Y.S. (2008) HPLC-MS/MS in Drug Metabolism and Pharmacokinetic Screening. Expert Opinion on Drug Metabolism \& Toxicology, 4, 93-101. http://dx.doi.org/10.1517/17425255.4.1.93

[3] Reshma, S., Kalpana, P. and Sandhya, B. (2011) RP-HPLC Method Development and Validation for the Analysis of Methylphenidate Hydrochloride in Pharmaceutical Dosage Form. Journal of Atomic and Molecular Sciences, 1, 64-73.

[4] Maria, C.F., Lepnardo, Z.M., Luciana, G.R. and Renata, P.L. (2009) Development and Validation of an LC-UV Method for Quantitation of 4-Bromo-2, 5-Dimethoxyamphetamine (DOB), 4-Bromo-2, 5-Dimethoxy Phenetylamine (2C-B), Methylphenidate, Fenproporex and Amfepramone. Chromatographia, 69, 143-148. http://dx.doi.org/10.1365/s10337-009-1044-9

[5] Pokkula, S., Thota, S., Kumar, V.R. and Nagabandi, V.K. (2014) Development and Validation of RP-HPLC Method for the Determination of Methylphenidate Hydrochloride in API. International Journal of PharmTech Research, 6, 462-467.

[6] Soldin, S.J., Chan, Y.P., Hill, B.M. and Swanson, J.M. (1982) Liquid-Chromatographic Analysis for Methylphenidate (Ritalin) in Serum. Journal of American Academy of Child and Adolescent Psychiatry, 21, 19-26.

[7] Sharon, M.P. (2012) Determination of Methylphenidate and Its Metabolite Ritalinic Acid in Urine by Liquid Chromatography/Tandem Mass Spectroscopy. Journal of Chromatography B, 881, 20-26.

[8] Secilir, A. (2013) Determination of Methylphenidate in Plasma and Saliva by Liquid Chromatography and Tandem Mass Spectroscopy. Journal of Chromatography B, 923, 22-28. http://dx.doi.org/10.1016/j.jchromb.2013.01.027

[9] Patrick, K.S., Ellington, K.R., Breese, G.R. and Kilts, C.D. (1985) Gas Chromatographic-Mass Spectrometric Analysis of Methylphenidate and p-Hydroxy Methylphenidate Using Deuterated Internal Standards. Journal of Chromatography A, 343, 329-338. http://dx.doi.org/10.1016/S0378-4347(00)84601-0

[10] Hotha, K.K., Reddy, S.P.K., Raju, V.K. and Ravindranath, L.K. (2013) Forced Degradation Studies: Practical Approach: Overview of Regulatory Guidance and Literature for the Drug Products and Drug Substances. International Research Journal of Pharmacy, 4, 78-85. http://dx.doi.org/10.7897/2230-8407.04517

[11] ICH Q2 (R1) (2005) Validation of Analytical Procedures: Text and Methodology.

[12] ICH Q1A (R2) (2000) Stability Testing of New Drug Substances and Products.

[13] ICH Guideline Q3A (R) (2002) Impurities in New Drug Substances.

[14] Meireles, B.A. and Pereira, V.L.P. (2013) Synthesis of Bio-Additives: Transesterification of Ethyl Acetate with Glycerol using Homogeneous or Heterogeneous Acid Catalysts. Journal of the Brazilian Chemical Society, 24, 17-25. http://dx.doi.org/10.1590/S0103-50532013000100004

[15] Nebel, B., Mittelbach, M. and Uray, G. (2008) Determination of the Composition of Acetyl Glycerol Mixtures by $1 \mathrm{H}$ NMR Followed by GC Investigation. Analytical Chemistry, 80, 8712-8716. http://dx.doi.org/10.1021/ac800706s

[16] Sutter, M., Dayoub, W., Métay, E., Raou, Y. and Lemaire, M. (2013) 1-O-Alkyl (Di) Glycerol Ethers Synthesis from Methyl Esters and Triglycerides by Two Pathways: Catalytic Reductive Alkylation and Transesterification/Reduction. Green Chemistry, 15, 786-797. http://dx.doi.org/10.1039/c3gc36907b

[17] Yu, H., Cornett, C., Larsen, J. and Hansen, S.H. (2010) Reaction between Drug Substances and Pharmaceutical Excipients: Formation of Esters between Cetirizine and Polyols. Journal of Pharmaceutical and Biomedical analysis, 53, 745-750. http://dx.doi.org/10.1016/j.jpba.2010.05.001 